\title{
Partial regularity for minimizers of variational integrals with discontinuous integrands
}

\author{
by
}

\author{
Christoph HAMBURGER
}

Mathematisches Institut der Universität Bonn, Beringstrasse 4, D-53115 Bonn, Germany.

\begin{abstract}
We prove partial regularity for vector-valued minimizers $u$ of the variational integral $\int[f(x, u, D u)+g(x, u)] d x$, where $f$ is strictly quasiconvex, of polynomial growth and continuous, but where $g$ is only a bounded Carathéodory function. We present an elementary proof for the special case of strict convexity and quadratic growth of $f(x, u, \cdot)$.
\end{abstract}

Key words: Partial regularity, minimizer, calculus of variations, quasiconvexity, discontinuous integrand, elementary proof.

RÉSUMÉ. - Nous donnons la preuve d'une régularité partielle pour des minimiseurs à valeurs vectorielles de l'intégrale variationnelle $\int[f(x, u, D u)+g(x, u)] d x$, où $f$ est strictement quasiconvexe, à croissance polynomiale et continue, mais où $g$ est seulement une fonction bornée de type Carathéodory. Nous présentons une preuve "élémentaire » pour un cas spécial de convexité stricte et de croissance quadratique de $f(x, u, \cdot)$. 


\section{INTRODUCTION}

We are interested in the regularity for the minimizers of the variational integral

$$
\mathcal{I}(u, \Omega)=\int_{\Omega} F(x, u(x), D u(x)) d x,
$$

defined for vector-valued functions $u: \Omega \rightarrow \mathbb{R}^{N}$, where $\Omega$ is a bounded domain in $\mathbb{R}^{n}, n \geq 2, N \geq 1$, and where $D u(x) \in \mathbb{R}^{N \times n}$ denotes the gradient of $u$ at the point $x \in \Omega$. The functional $\mathcal{I}(u, \Omega)$ is well-defined for $u \in W^{1, q}\left(\Omega, \mathbb{R}^{N}\right)$, with exponent $q \geq 2$, if we admit as integrands Carathéodory functions $F(x, u, P): \bar{\Omega} \times \mathbb{R}^{N} \times \mathbb{R}^{N \times n} \rightarrow \mathbb{R}$ of polynomial growth in $P$, i.e. functions which are measurable in $x$, continuous in $(u, P)$ and which satisfy the growth condition

$$
|F(x, u, P)| \leq \Gamma\left(1+|P|^{q}\right) .
$$

DefinItion 1.1. - We call $u \in W^{1, q}\left(\Omega, \mathbb{R}^{N}\right)$ a minimizer of $\mathcal{I}$ if

$$
\mathcal{I}(u, \operatorname{supp} \varphi) \leq \mathcal{I}(u+\varphi, \operatorname{supp} \varphi) \quad \text { for every } \quad \varphi \in C_{0}^{\infty}\left(\Omega, \mathbb{R}^{N}\right) .
$$

The problem of regularity for the minimizers of $\mathcal{I}$ has been intensively investigated over the last 10 years [G-G2] [E1] [E-G1] [F-H] [G-M] [A-F] [G3]. In the thus established partial regularity theory for vector-valued minimizers one requires the integrand $F(x, u, P)$ to be of class $C^{2}$ in $P$, strictly quasiconvex, and to satisfy the coercivity and growth conditions

$\gamma|P|^{q} \leq F(x, u, P) \leq \Gamma\left(1+|P|^{q}\right), \quad\left|F_{P P}(x, u, P)\right| \leq \Gamma\left(1+|P|^{q-2}\right)$.

In addition one assumes that $F(x, u, P)$ be Hölder continuous in $(x, u)$. By partial regularity of a minimizer $u \in W^{1, q}\left(\Omega, \mathbb{R}^{N}\right)$ we mean Hölder continuity of the gradient $D u$ except possibly on a closed set of Lebesgue measure zero.

It is the aim of this paper to extend the partial regularity theory to variational integrals with possibly discontinuous integrands of the form

$$
F(x, u, P)=f(x, u, P)+g(x, u),
$$


where $f(x, u, P)$ as well as $f_{P}(x, u, P)$ are Hölder continuous in $(x, u)$, but where $g(x, u)$ is only measurable in $x$ and Hölder continuous in $u$.

Furthermore, for the special case when the integrand $F(x, u, P)$ is of quadratic growth $(q=2)$ and strictly convex in $P$,

$$
F_{P P}(x, u, P) \cdot(\zeta, \zeta) \geq \gamma|\zeta|^{2}
$$

for some $\gamma>0$ and all $\zeta \in \mathbb{R}^{N \times n}$, we present an elementary proof based solely on elliptic estimates for linear systems of P.D.E. with constant coefficients.

The general idea in the proof of partial regularity is to compare the minimizer with a solution of a constant coefficient operator equation, for which standard elliptic estimates are available. For the direct proof this comparison is carried out on an arbitrary ball under a Dirichlet boundary condition; for the indirect proof it is shown that a sequence of blow-up functions $w_{m} \in W^{1,2}\left(B, \mathbb{R}^{N}\right)$, rescaled to the unit ball $B$, converges weakly to such a solution.

We start with a short account of the development of the partial regularity theory for vector-valued minimizers, see also [G2]. Assuming quadratic growth and strict convexity in $P$ for the integrand $F(x, u, P)$ and using a direct argument, M. Giaquinta and E. Giusti [G-G2] proved partial regularity for minimizers $u \in W^{1,2}\left(\Omega, \mathbb{R}^{N}\right)$.

The next step came with the work of L. C. Evans [E1]. For integrands $F(P)$ depending solely on the variable $P$, he gave an indirect proof of partial regularity for minimizers replacing strict convexity by strict quasiconvexity. Partial regularity for minimizers was shown to hold also true if the integrand $F(x, u, P)$ depends on all the variables, provided that one assumes, besides strict quasiconvexity, that $F$ be coercive. Various proofs were given for this result, some direct [G-M], some indirect [F-H], and some allowing for a weaker form of coercivity [Ho], or assuming no growth condition for $F_{P P}$ [A-F] [G3]. In all cases a Caccioppoli inequality holds for the minimizer $u$, which gives rise to a reverse Hölder inequality with increasing supports for $D u-P_{0}$, for constant $P_{0} \in \mathbb{R}^{N \times n}$. The direct proofs depend on this reverse Hölder inequality, and also the indirect proofs make use of the Caccioppoli inequality.

It was again L. C. Evans who in collaboration with R. F. Gariepy [E-G1] showed that, for the case of an integrand $F(P)$, a Caccioppoli inequality is not needed. Instead they devised a technique for establishing convergence $w_{m} \rightarrow w$ in $W_{\text {loc }}^{1,2}$ of a sequence of blow-up functions $w_{m} \in W^{1,2}\left(B, \mathbb{R}^{N}\right)$, 
which are known to converge only weakly. A key estimate for this procedure is

$$
\limsup _{m \rightarrow \infty}\left(\mathcal{I}_{m}\left(w_{m}, B_{r}\right)-\mathcal{I}_{m}\left(w, B_{r}\right)\right) \leq 0
$$

where $\mathcal{I}_{m}\left(\cdot, B_{r}\right)$ are suitably rescaled variational integrals on a ball of radius $r \leq 1$, or, equivalently,

$$
\limsup _{m \rightarrow \infty} \mathcal{I}_{m}\left(w_{m}, B_{r}\right) \leq \mathcal{I}_{0}\left(w, B_{r}\right)
$$

where $\mathcal{I}_{0}$ is the limit functional of the sequence $\mathcal{I}_{m}$.

In the present paper we pursue the ideas of L. C. Evans and R. F. Gariepy further and apply them to the general case of an integrand $F(x, u, P)$ depending on all the variables. While L. C. Evans and R. F. Gariepy were able to use strict quasiconvexity directly in proving convergence in $W_{\text {loc }}^{1,2}$ of the sequence of blow-up functions $w_{m}$ satisfying (1.2), we instead rely on the following technique.

First we show that the estimate (1.2) is also valid for the case of an integrand $F(x, u, P)$ where both $u \rightarrow F(x, u, P)$ and $(x, u) \rightarrow$ $F_{P}(x, u, P)$ are Hölder continuous. Thus we may admit discontinuities of $x \rightarrow F(x, u, P)$ that do not propagate to $F_{P}(x, u, P)$, as for the above mentioned class of integrands of the form (1.1). We point out that our continuity assumption for the integrand stands in marked contrast to the standard hypothesis that $(x, u) \rightarrow F(x, u, P)$ be Hölder continuous without imposing any condition on $F_{P}(x, u, P)$.

The estimate (1.2) involves a sequence of functionals $\mathcal{I}_{m}$ rather than a fixed functional. We can however express the rescaled functionals $\mathcal{I}_{m}$ by means of suitably chosen functions $Y_{m}$ as a fixed functional

$$
\mathcal{I}_{m}\left(w_{m}, B_{r}\right)=\int_{B_{r}} G\left(Y_{m}\right) \cdot\left(D w_{m}, D w_{m}\right) d z
$$

whose integrand $G(Y) \cdot(\zeta, \zeta)$ is quadratic in $\zeta$. Moreover, the functions $Y_{m}$ converge in $L^{2}$ to a constant function $Y_{0}$. Thus we have the estimate

$$
\begin{gathered}
\limsup _{m \rightarrow \infty} \int_{B_{r}} G\left(Y_{m}\right) \cdot\left(D w_{m}, D w_{m}\right) d z \\
\leq \int_{B_{r}} G\left(Y_{0}\right) \cdot(D w, D w) d z .
\end{gathered}
$$


We now distinguish two cases. In the special case that $G(Y)$ is bounded and uniformly positive,

$$
G(Y) \cdot(\zeta, \zeta) \geq \gamma|\zeta|^{2}
$$

which in terms of the original integrand $F(x, u, P)$ amounts to quadratic growth and strict convexity in the variable $P$, we deduce with the help of a simple convergence lemma that $w_{m} \rightarrow w$ in $W_{\text {loc }}^{1,2}$. It then follows easily that $w$ solves a linear elliptic system with constant coefficients. We thus obtain an elementary partial regularity proof for minimizers in the spirit of L. C. Evans and R. F. Gariepy [E-G1] which does not use a Caccioppoli inequality or the involved higher integrability theorem of F. W. Gehring, M. Giaquinta and G. Modica.

This elementary proof does not generalize to minimizers of quasiconvex functionals, as the convergence lemma fails in this context-we illustrate this fact by a counterexample. We note, however, that such an elementary type of partial regularity proof works for solutions of nonlinear elliptic systems [H].

In the general case we assume $F(x, u, P)$ to be strictly quasiconvex, coercive and of polynomial growth in $P$ with exponent $q \geq 2$. By virtue of the higher integrability theorem, we then deduce from a Caccioppoli inequality a reverse Hölder inequality for $D u-P_{0}$, for constant $P_{0} \in \mathbb{R}^{N \times n}$. This leads to a uniform bound of $\left\{D w_{m}\right\}$ in $L_{\text {loc }}^{2+\varepsilon}$, for some $\varepsilon>0$, which allows us to prove that $w$ is a weak solution of a linear elliptic system with constant coefficients. Knowing the regularity of $w$, we can finally deduce from (1.3) that $w_{m} \rightarrow w$ in $W_{\text {loc }}^{1,2}$. In this manner we establish partial regularity for minimizers of quasiconvex variational integrals with discontinuous integrands of the form (1.1).

For the integrand $F(x, u, P): \bar{\Omega} \times \mathbb{R}^{N} \times \mathbb{R}^{N \times n} \rightarrow \mathbb{R}$ we shall assume the following hypotheses, for an exponent $q \geq 2$.

Hypothesis H1. - We suppose that $F(x, u, P)$ is of class $C^{2}$ in $P$, and we assume that $F_{P P}$ is continuous and of polynomial growth:

$$
\left|F_{P P}(x, u, P)\right| \leq \Gamma\left(1+|P|^{q-2}\right) .
$$

Hypothesis H2. - We suppose that $u \rightarrow F(x, u, P)$ and $(x, u) \rightarrow$ $F_{P}(x, u, P)$ are Hölder continuous in the sense that

$$
\begin{gathered}
|F(x, u, P)-F(x, v, P)| \leq\left(1+|P|^{q}\right) \omega(|u|,|u-v|), \\
\left|F_{P}(x, u, P)-F_{P}(y, v, P)\right| \leq\left(1+|P|^{q-1}\right) \omega(|u|,|x-y|+|u-v|),
\end{gathered}
$$


for all $x, y \in \bar{\Omega}, u, v \in \mathbb{R}^{N}, P \in \mathbb{R}^{N \times n}$. Here $\omega(s, t)=K(s) \min \left(t^{\delta}, 1\right)$, for $0<\delta \leq 1$ and for a nondecreasing function $K(s)$; note that $t \rightarrow \omega(s, t)$, for fixed $s$, is concave and bounded.

HYPOTHESIS H3. - We suppose that $F(x, u, P)$ is uniformly strictly quasiconvex

$$
\begin{aligned}
& \gamma \int_{\mathbb{R}^{n}}\left(|D \varphi|^{2}+|D \varphi|^{q}\right) d x \\
& \quad \leq \int_{\mathbb{R}^{n}}\left(F\left(x_{0}, u_{0}, P_{0}+D \varphi\right)-F\left(x_{0}, u_{0}, P_{0}\right)\right) d x,
\end{aligned}
$$

for some $\gamma>0$ and all $\left(x_{0}, u_{0}, P_{0}\right) \in \Omega \times \mathbb{R}^{N} \times \mathbb{R}^{N \times n}$, and $\varphi \in$ $C_{0}^{\infty}\left(\mathbb{R}^{n}, \mathbb{R}^{N}\right)$.

HyPOTHESIS H4. - We suppose that

$$
F(x, u, P) \geq \tilde{F}(P)
$$

for all $(x, u, P) \in \bar{\Omega} \times \mathbb{R}^{N} \times \mathbb{R}^{N \times n}$, where $\tilde{F}(P)$ is a function which is strictly quasiconvex at $P_{0}=0$ :

$$
\gamma \int_{\mathbb{R}^{n}}|D \varphi|^{q} d x \leq \int_{\mathbb{R}^{n}}(\tilde{F}(D \varphi)-\tilde{F}(0)) d x
$$

for some $\gamma>0$ and for all $\varphi \in C_{0}^{\infty}\left(\mathbb{R}^{n}, \mathbb{R}^{N}\right)$.

Remark. - Hypothesis $\mathrm{H} 4$ is fulfilled for example if $F(x, u, P)$ is coercive

$$
F(x, u, P) \geq \gamma|P|^{q} .
$$

Our main result is contained in the following theorem.

THEOREM 1.1. - Let the integrand $\mathrm{F}$ satisfy Hypotheses $\mathrm{HI}, \mathrm{H} 2, \mathrm{H} 3$ and $H 4$, with exponent $q \geq 2$, and let $u \in W^{1, q}\left(\Omega, \mathbb{R}^{N}\right)$ be a minimizer for the variational integral $\mathcal{I}$.

Then there exist an open set $\Omega_{0} \subset \Omega$, whose complement has Lebesgue measure zero, and a positive number $\alpha_{0}<\frac{1}{2} \delta$ such that the gradient $D u$ is locally Hölder continuous in $\Omega_{0}$, with any exponent $0<\alpha<\alpha_{0}$.

$$
u \in C^{1, \alpha}\left(\Omega_{0}, \mathbb{R}^{N}\right) \quad \text { and } \quad \mathcal{L}^{n}\left(\Omega \backslash \Omega_{0}\right)=0 .
$$

Moreover, the regular set is characterized by

$$
\begin{aligned}
& \Omega_{0}=\left\{x_{0} \in \Omega: \sup _{r>0}\left(\left|u_{x_{0}, r}\right|+\left|D u_{x_{0}, r}\right|\right)<\infty\right. \\
& \left.\quad \text { and } \liminf _{r \rightarrow 0+} f_{B_{r}\left(x_{0}\right)}\left|D u-D u_{x_{0}, r}\right|^{q} d x=0\right\} .
\end{aligned}
$$


Remark. - We have

$$
\alpha_{0}=\frac{\varepsilon_{1}}{1+\varepsilon_{1}} \frac{\delta}{2}
$$

where $\varepsilon_{1}$ is the higher integrability constant appearing in Theorem 4.2.

We also state a special case of Theorem 1.1, for which we provide an elementary proof.

Hypothesis $\mathrm{H}^{*}$. - We suppose that $F(x, u, P)$ is uniformly strictly convex in $P$

$$
F_{P P}(x, u, P) \cdot(\zeta, \zeta) \geq \gamma|\zeta|^{2}
$$

for some $\gamma>0$ and all $(x, u, P) \in \bar{\Omega} \times \mathbb{R}^{N} \times \mathbb{R}^{N \times n}$, and $\zeta \in \mathbb{R}^{N \times n}$.

THEOREM 1.2. - Let the integrand $F$ satisfy Hypotheses $H 1, H 2$ and $H 3 *$, with exponent $q=2$, and let $u \in W^{1,2}\left(\Omega, \mathbb{R}^{N}\right)$ be a minimizer for the variational integral $\mathcal{I}$.

Then there exists an open set $\Omega_{0} \subset \Omega$, whose complement has Lebesgue measure zero, such that the gradient $D u$ is locally Hölder continuous in $\Omega_{0}$, with any exponent $0<\alpha<\frac{1}{2} \delta$.

\section{A DECAY ESTIMATE FOR THE EXCESS}

In the sequel all constants $c$ may depend on the data $n, N, q, \gamma, \Gamma, \delta, K(\cdot)$ and on the number $L$ from the proof of Proposition 2.1. The Landau symbol $o$ (1) stands for any quantity for which $\lim _{m \rightarrow \infty} o(1)=0$. We write $B_{r}\left(x_{0}\right)=\left\{x \in \mathbb{R}^{n}:\left|x-x_{0}\right|<r\right\}$, and $B=B_{1}(0)$ for the unit ball. We denote the mean value by

$$
f_{x_{0}, r}=f_{B_{r}\left(x_{0}\right)} f d x=\frac{1}{\mathcal{L}^{n}\left(B_{r}\left(x_{0}\right)\right)} \int_{B_{r}\left(x_{0}\right)} f d x .
$$

In this section we assume Hypotheses $\mathrm{H} 1, \mathrm{H} 2$, and either of $\mathrm{H} 3$ or $\mathrm{H} 3$ *, with $q \geq 2$. We let $u \in W^{1, q}\left(\Omega, \mathbb{R}^{N}\right)$ be a minimizer for the functional $\mathcal{I}$. We choose any positive number $\alpha<\alpha_{0}$, where $\alpha_{0}$ is given by (1.4), and we define the excess of $D u$ on the ball $B_{r}\left(x_{0}\right) \subset \subset \Omega$ :

$U\left(x_{0}, r\right)=r^{2 \alpha}+f_{B_{r}\left(x_{0}\right)}\left|D u-D u_{x_{0}, r}\right|^{2} d x+f_{B_{r}\left(x_{0}\right)}\left|D u-D u_{x_{0}, r}\right|^{q} d x$

(in the case of Theorem 1.2 we choose any positive number $\alpha<\frac{1}{2} \delta$ ).

Theorems 1.1 and 1.2 follow in a routine way from the following proposition, see [G1, pp. 198-199] [E1, Section 7] [F-H, Section 6]. 
Proposition 2.1. - Let $L>0$ and $\tau \in] 0,1[$ be given. Then there exist positive constants $c_{1}(L)$ and $\varepsilon(L, \tau)$ such that if $B_{r}\left(x_{0}\right) \subset \subset \Omega, \quad\left|u_{x_{0}, r}\right| \leq L, \quad\left|D u_{x_{0}, r}\right| \leq L \quad$ and $\quad U\left(x_{0}, r\right) \leq \varepsilon(L, \tau)$ then

$$
U\left(x_{0}, \tau r\right) \leq c_{1}(L) \tau^{2 \alpha} U\left(x_{0}, r\right) .
$$

Proof. - We determine the constant $c_{1}(L)$ later on. If the proposition were not true then there would exist a sequence of balls $B_{m}=B_{r_{m}}\left(x_{m}\right) \subset \subset \Omega$ such that

$$
\begin{gathered}
\left|u_{x_{m}, r_{m}}\right| \leq L, \quad\left|D u_{x_{m}, r_{m}}\right| \leq L, \\
U\left(x_{m}, r_{m}\right)=\lambda_{m}^{2} \rightarrow 0 \quad \text { as } m \rightarrow \infty,
\end{gathered}
$$

but

$$
U\left(x_{m}, \tau r_{m}\right)>c_{1}(L) \tau^{2 \alpha} \lambda_{m}^{2}
$$

We set

$$
u_{m}=u_{x_{m}, r_{m}}, \quad P_{m}=D u_{x_{m}, r_{m}}
$$

and we define the rescaled functions

$$
w_{m}(z)=\frac{u\left(x_{m}+r_{m} z\right)-u_{m}-r_{m} P_{m} \cdot z}{r_{m} \lambda_{m}}
$$

for each $m$ and for all $z \in B$. Notice that

$$
\begin{gathered}
D w_{m}(z)=\frac{D u\left(x_{m}+r_{m} z\right)-P_{m}}{\lambda_{m}}, \\
\left(w_{m}\right)_{0,1}=0, \quad\left(D w_{m}\right)_{0,1}=0 .
\end{gathered}
$$

Then (2.1) and (2.2) become

$$
\begin{gathered}
\left|u_{m}\right| \leq L, \quad\left|P_{m}\right| \leq L, \\
\lambda_{m}^{-2} r_{m}^{2 \alpha}+f_{B}\left|D w_{m}\right|^{2} d z+\lambda_{m}^{q-2} f_{B}\left|D w_{m}\right|^{q} d z=1, \\
c_{1}(L) \tau^{2 \alpha}<\lambda_{m}^{-2} r_{m}^{2 \alpha} \tau^{2 \alpha}+f_{B_{\tau}}\left|D w_{m}-\left(D w_{m}\right)_{0, \tau}\right|^{2} d z \\
+\lambda_{m}^{q-2} f_{B_{\tau}}\left|D w_{m}-\left(D w_{m}\right)_{0, \tau}\right|^{q} d z .
\end{gathered}
$$


From (2.7) we immediately have

$$
\lambda_{m}^{-1} r_{m}^{\alpha} \leq 1, \quad\left\|D w_{m}\right\|_{L^{2}(B)} \leq c, \quad \lambda_{m}^{(q-2) / q}\left\|D w_{m}\right\|_{L^{q}(B)} \leq c .
$$

Since $\lambda_{m} \rightarrow 0$ as $m \rightarrow \infty$, and $\alpha<\frac{1}{2} \delta$, it follows from (2.9) that

$$
r_{m} \rightarrow 0 \quad \text { and } \quad \lambda_{m}^{-1} r_{m}^{\frac{1}{2} \delta} \rightarrow 0 \quad \text { as } m \rightarrow \infty .
$$

We infer from (2.5) and (2.9) by the Poincaré inequality that

$$
\left\|w_{m}\right\|_{L^{2}(B)} \leq c, \quad \lambda_{m}^{(q-2) / q}\left\|w_{m}\right\|_{L^{q}(B)} \leq c .
$$

It follows from (2.9), (2.11) and (2.6) that, on passing to a subsequence and relabelling, we have

$$
\begin{array}{lll}
D w_{m} \rightarrow D w & \text { weakly in } L^{2}\left(B, \mathbb{R}^{N \times n}\right), & \\
w_{m} \rightarrow w & \text { in } L^{2}\left(B, \mathbb{R}^{N}\right), & \\
\lambda_{m}^{(q-2) / q} D w_{m} \rightarrow 0 & \text { weakly in } L^{q}\left(B, \mathbb{R}^{N \times n}\right) & (\text { for } q>2), \\
\lambda_{m}^{(q-2) / q} w_{m} \rightarrow 0 & \text { in } L^{q}\left(B, \mathbb{R}^{N}\right) & (\text { for } q>2), \\
\lambda_{m} D w_{m} \rightarrow 0 & \text { in } L^{2}\left(B, \mathbb{R}^{N \times n}\right), & \\
\left(x_{m}, u_{m}, P_{m}\right) \rightarrow\left(x_{0}, u_{0}, P_{0}\right) & \text { in } \bar{\Omega} \times \mathbb{R}^{N} \times \mathbb{R}^{N \times n} . &
\end{array}
$$

We let $\mathcal{M}(B)$ denote the space of real-valued Radon measures on $B$ with finite mass. We define the positive measures

$$
\mu_{m}=1+\left|D w_{m}\right|^{2}+|D w|^{2}+\lambda_{m}^{q-2}\left|D w_{m}\right|^{q},
$$

which are uniformly bounded in $\mathcal{M}(B)$ by (2.9). Hence there exists a further subsequence, which we again relabel and index by $m$, and $\mu \in \mathcal{M}(B)$ such that

$$
\mu_{m} \stackrel{*}{\rightarrow} \mu \quad \text { in the weak }{ }^{*} \text {-topology of } \mathcal{M}(B) .
$$

Since $\mu_{m} \geq 0$, this implies that

$$
\limsup _{m \rightarrow \infty} \mu_{m}(K) \leq \mu(K)
$$

for every compact set $K \subset B$, i.e. the functional $\mu \rightarrow \mu(K)$ is sequentially weakly* upper semicontinuous on nonnegative measures. 
Proof. - Given $\varepsilon>0$, there exist an open set $V \subset B$, such that $K \subset V$ and $\mu(V)<\mu(K)+\varepsilon$, and a function $f \in C(B)$ with supp $f \subset \subset V$, $0 \leq f \leq 1$ and $f \equiv 1$ on $K$, see [R, Thms. 2.14(c) and 2.12]. By weak*-convergence

$$
\mu_{m}(K) \leq \int_{B} f d \mu_{m} \rightarrow \int_{B} f d \mu \leq \mu(V)<\mu(K)+\varepsilon
$$

and (2.14) follows.

Because $\mu(B)<\infty$, we have $\mu\left(\partial B_{r}\right)=0$ for all but at most countably many $r \in] 0,1[$.

Now suppose that we can show that $w \in W^{1,2}\left(B, \mathbb{R}^{N}\right)$ is a weak solution of the following linear system of partial differential equations with constant coefficients:

$$
\operatorname{div}\left(F_{P P}\left(x_{0}, u_{0}, P_{0}\right) \cdot D w\right)=0
$$

We infer from $\mathrm{H} 1$ and (2.6) that

$$
\left|F_{P P}\left(x_{0}, u_{0}, P_{0}\right)\right| \leq c,
$$

and from $\mathrm{H} 3$ or $\mathrm{H} 3 *$ that (2.15) is elliptic, see [E2, Ch. 3]:

$F_{P P}\left(x_{0}, u_{0}, P_{0}\right) \cdot(\eta \otimes \xi, \eta \otimes \xi) \geq \gamma|\eta \otimes \xi|^{2} \quad$ for all $\eta \in \mathbb{R}^{N}, \xi \in \mathbb{R}^{n}$.

Hence, from the theory of linear elliptic systems [G1, Thm. 2.1, Remarks 2.2, 2.3, pp. 77-79], (2.5) and (2.9), we conclude that $w \in$ $C^{\infty}\left(B, \mathbb{R}^{N}\right)$ and

$$
f_{B_{\tau}}\left|D w-D w_{0, \tau}\right|^{2} d z \leq c_{2}(L) \tau^{2}
$$

On the other hand, if we also know that

$$
\begin{gathered}
D w_{m} \rightarrow D w \quad \text { in } L_{\mathrm{loc}}^{2}\left(B, \mathbb{R}^{N \times n}\right), \\
\lambda_{m}^{(q-2) / q} D w_{m} \rightarrow 0 \quad \text { in } L_{\mathrm{loc}}^{q}\left(B, \mathbb{R}^{N \times n}\right) \quad(\text { for } q>2),
\end{gathered}
$$

then it would follow from (2.8) and (2.9) that

$$
c_{1}(L) \tau^{2 \alpha} \leq \tau^{2 \alpha}+f_{B_{\tau}}\left|D w-D w_{0, \tau}\right|^{2} d z .
$$

If we now choose $c_{1}(L)>1+c_{2}(L)$, we obtain a contradiction to (2.16). This proves the proposition. 
The remainder of this work is devoted to proving (2.15), (2.17) and (2.18). To this end we set

$$
\begin{aligned}
& F_{m}(z, w, R) \\
& =\lambda_{m}^{-2}\left(F\left(x_{m}+r_{m} z, u_{m}+r_{m} P_{m} \cdot z+r_{m} \lambda_{m} w, P_{m}+\lambda_{m} R\right)\right. \\
& -F\left(x_{m}+r_{m} z, u_{m}+r_{m} P_{m} \cdot z+r_{m} \lambda_{m} w, P_{m}\right) \\
& \left.-F_{P}\left(x_{m}+r_{m} z, u_{m}+r_{m} P_{m} \cdot z+r_{m} \lambda_{m} w, P_{m}\right) \cdot \lambda_{m} R\right) \\
& =\int_{0}^{1}(1-s) F_{P P}\left(x_{m}+r_{m} z, u_{m}+r_{m} P_{m} \cdot z+r_{m} \lambda_{m} w, P_{m}+s \lambda_{m} R\right) \\
& \times(R, R) d s .
\end{aligned}
$$

By $\mathrm{H} 1$ and (2.6), we note the estimate

$$
\left|F_{m}(z, w, R)\right| \leq c\left(|R|^{2}+\lambda_{m}^{q-2}|R|^{q}\right),
$$

and we also define the functionals

$$
\begin{gathered}
\mathcal{I}_{m}(w, U)=\int_{U} F_{m}(z, w, D w) d z \\
\mathcal{I}_{0}(w, U)=\frac{1}{2} \int_{U} F_{P P}\left(x_{0}, u_{0}, P_{0}\right) \cdot(D w, D w) d z
\end{gathered}
$$

for $w \in W^{1, q}\left(B, \mathbb{R}^{N}\right)$ and measurable subsets $U \subset B$.

We next show that $w_{m}$ is, to order zero as $m \rightarrow \infty$, a minimizer for $\mathcal{I}_{m}$ :

Lemma 2.1. - For $\varphi \in W_{0}^{1, q}\left(B, \mathbb{R}^{N}\right)$ we have

$$
\mathcal{I}_{m}\left(w_{m}, \operatorname{supp} \varphi\right) \leq \mathcal{I}_{m}\left(w_{m}+\varphi, \operatorname{supp} \varphi\right)+o(1)
$$

where

$$
o(1)=c\left(1+\|\varphi\|_{W^{1,2}}^{2}\right) \lambda_{m}^{-1} r_{m}^{\frac{1}{2} \delta} .
$$

Proof. - On rescaling we obtain from the minimality of $u$ that

$$
\begin{array}{rl}
\int_{B} & F\left(x_{m}+r_{m} z, u_{m}+r_{m} P_{m} \cdot z+r_{m} \lambda_{m} w_{m}, P_{m}+\lambda_{m} D w_{m}\right) d z \\
\leq \int_{B} F\left(x_{m}+r_{m} z, u_{m}+r_{m} P_{m} \cdot z+r_{m} \lambda_{m}\left(w_{m}+\varphi\right)\right. \\
\left.\quad P_{m}+\lambda_{m}\left(D w_{m}+D \varphi\right)\right) d z
\end{array}
$$

Vol. 13, n 3-1996. 
and it follows that

$$
\begin{aligned}
& \mathcal{I}_{m}\left(w_{m}, B\right)-\mathcal{I}_{m}\left(w_{m}+\varphi, B\right) \\
& \leq \lambda_{m}^{-2} \int_{B}\left(F\left(x_{m}+r_{m} z, u_{m}+r_{m} P_{m} \cdot z+r_{m} \lambda_{m}\left(w_{m}+\varphi\right), P_{m}\right)\right. \\
& \left.\quad-F\left(x_{m}+r_{m} z, u_{m}, P_{m}\right)\right) d z \\
& -\lambda_{m}^{-2} \int_{B}\left(F\left(x_{m}+r_{m} z, u_{m}+r_{m} P_{m} \cdot z+r_{m} \lambda_{m} w_{m}, P_{m}\right)\right. \\
& \left.\quad-F\left(x_{m}+r_{m} z, u_{m}, P_{m}\right)\right) d z \\
& +\lambda_{m}^{-1} \int_{B}\left(F_{P}\left(x_{m}+r_{m} z, u_{m}+r_{m} P_{m} \cdot z+r_{m} \lambda_{m}\left(w_{m}+\varphi\right), P_{m}\right)\right. \\
& \left.\quad-F_{P}\left(x_{m}+r_{m} z, u_{m}, P_{m}\right)\right) \cdot D w_{m} d z \\
& -\lambda_{m}^{-1} \int_{B}\left(F_{P}\left(x_{m}+r_{m} z, u_{m}+r_{m} P_{m} \cdot z+r_{m} \lambda_{m} w_{m}, P_{m}\right)\right. \\
& \left.\quad-F_{P}\left(x_{m}+r_{m} z, u_{m}, P_{m}\right)\right) \cdot D w_{m} d z \\
& +\lambda_{m}^{-1} \int_{B}\left(F_{P}\left(x_{m}+r_{m} z, u_{m}+r_{m} P_{m} \cdot z+r_{m} \lambda_{m}\left(w_{m}+\varphi\right), P_{m}\right)\right. \\
& \left.\quad-F_{P}\left(x_{m}, u_{m}, P_{m}\right)\right) \cdot D \varphi d z \\
& =(\mathrm{I})+(\mathrm{II})+(\mathrm{III})+(\mathrm{IV})+(\mathrm{V}) .
\end{aligned}
$$

By virtue of Hypothesis $\mathrm{H} 2$, we estimate the term (I) as follows (using (2.6), Jensen's inequality in combination with the concavity of $\omega(L, \cdot)$, (2.11) and the inequality $\left.\omega(L, t) \leq c t^{\delta}\right)$ :

$$
\begin{aligned}
& (\mathrm{I}) \leq c\left(1+\left|P_{m}\right|^{q}\right) \lambda_{m}^{-2} f_{B} \omega\left(\left|u_{m}\right|, r_{m}\left|P_{m}\right|+r_{m} \lambda_{m}\left|w_{m}+\varphi\right|\right) d z \\
& \leq c \lambda_{m}^{-2} \omega\left(L, r_{m} L+r_{m} \lambda_{m} f_{B}\left|w_{m}+\varphi\right| d z\right) \leq c \lambda_{m}^{-2} r_{m}^{\delta}\left(1+\|\varphi\|_{L^{2}}^{\delta}\right) .
\end{aligned}
$$

For the term (V), we first use Hölder's inequality and the boundedness of $\omega(L, \cdot)$.

$$
\begin{aligned}
(\mathrm{V}) & \leq c \lambda_{m}^{-1} f_{B} \omega\left(\left|u_{m}\right|, r_{m}+r_{m}\left|P_{m}\right|+r_{m} \lambda_{m}\left|w_{m}+\varphi\right|\right)|D \varphi| d z \\
& \leq c \lambda_{m}^{-1}\left(f_{B} \omega\left(L, r_{m}+r_{m} L+r_{m} \lambda_{m}\left|w_{m}+\varphi\right|\right) d z\right)^{\frac{1}{2}}\|\varphi\|_{W^{1,2}} \\
& \leq c \lambda_{m}^{-1} r_{m}^{\frac{1}{2} \delta}\left(1+\|\varphi\|_{L^{2}}^{\frac{1}{2} \delta}\right)\|\varphi\|_{W^{1,2}} .
\end{aligned}
$$

The estimates of (II), (III) and (IV) are similar. 
We end this section with introducing some further notation. We first define the set

$$
\mathfrak{Y}=\left\{(x, u, P, Q) \in \bar{\Omega} \times \mathbb{R}^{N} \times \mathbb{R}^{N \times n} \times \mathbb{R}^{N \times n}:|P| \leq L\right\} \subset \mathbb{R}^{s},
$$

for $s=n+N+2 N n$, and the function spaces

$$
L^{p}(B, \mathfrak{Y})=\left\{Y \in L^{p}\left(B, \mathbb{R}^{s}\right): Y(z) \in \mathfrak{Y} \quad \text { for a.e. } z \in B\right\},
$$

for $p>1$.

We next define the symmetric bilinear form $G(Y)$ on $\mathbb{R}^{N \times n}$, for $Y=(x, u, P, Q) \in \mathfrak{Y}$, by

$$
G(Y)=G(x, u, P, Q)=\int_{0}^{1}(1-s) F_{P P}(x, u, P+s Q) d s .
$$

By Hypothesis H1, the bilinear form $G(Y)$ depends continuously on $Y \in \mathfrak{Y}$ and satisfies the growth condition

$$
|G(Y)| \leq c\left(1+|Q|^{q-2}\right)
$$

for all $Y=(x, u, P, Q) \in \mathfrak{Y}$.

We also define the corresponding quadratic functional $\mathcal{G}_{r}$, with $0<r<1$, by

$$
\mathcal{G}_{r}(Y, w)=\int_{B_{r}} G(Y) \cdot(D w, D w) d z
$$

for $Y \in L^{q}(B, \mathfrak{Y})$ and $w \in W^{1, q}\left(B, \mathbb{R}^{N}\right)$.

By (2.19) we observe that

(2.24) $F_{m}(z, w, R)$

$$
=G\left(x_{m}+r_{m} z, u_{m}+r_{m} P_{m} \cdot z+r_{m} \lambda_{m} w, P_{m}, \lambda_{m} R\right) \cdot(R, R),
$$

and hence

$$
F_{m}\left(z, w_{m}, D w_{m}\right)=G\left(Y_{m}\right) \cdot\left(D w_{m}, D w_{m}\right)
$$

for the functions $Y_{m} \in L^{q}(B, \mathfrak{Y})$ defined by

$$
=\left(x_{m}+r_{m} z, u_{m}+r_{m} P_{m} \cdot z+r_{m} \lambda_{m} w_{m}(z), P_{m}, \lambda_{m} D w_{m}(z)\right) .
$$


By (2.10) and (2.12), we notice the convergence

$$
Y_{m} \rightarrow Y_{0} \quad \text { in } L^{2}(B, \mathfrak{Y})
$$

to the constant function with value

$$
Y_{0}=\left(x_{0}, u_{0}, P_{0}, 0\right) \in \mathfrak{Y} .
$$

Now (2.25) and (2.28) imply that

$$
\mathcal{I}_{m}\left(w_{m}, B_{r}\right)=\mathcal{G}_{r}\left(Y_{m}, w_{m}\right), \quad \mathcal{I}_{0}\left(\cdot, B_{r}\right)=\mathcal{G}_{r}\left(Y_{0}, \cdot\right)
$$

\section{AN ELEMENTARY PROOF IN A SPECIAL CASE}

In this section we assume Hypotheses $\mathrm{H} 1, \mathrm{H} 2$ and $\mathrm{H} 3^{*}$, with $q=2$. We note the estimates

$$
G(Y) \cdot(\zeta, \zeta) \geq \frac{\gamma}{2}|\zeta|^{2}, \quad|G(Y)| \leq \frac{\Gamma}{2} \quad \text { for } Y \in \mathfrak{Y}, \zeta \in \mathbb{R}^{N \times n}
$$

Now we fix some $r \in] 0,1\left[\right.$ with $\mu\left(\partial B_{r}\right)=0$ (recall that a.e. $\left.r \in\right] 0,1[$ satisfies this condition). We let $0<s<r$ and $\zeta \in C_{0}^{\infty}\left(B_{r}\right)$ be a cut-off function with $0 \leq \zeta \leq 1$ and $\zeta \equiv 1$ on $B_{s}$. We then set $\varphi=\zeta\left(w-w_{m}\right) \in W_{0}^{1,2}\left(B, \mathbb{R}^{N}\right)$ in $(2.21)$, for which

$$
D \varphi=\zeta\left(D w-D w_{m}\right)+\left(w-w_{m}\right) \otimes D \zeta .
$$

Using (2.20) and (2.13), this yields

$$
\begin{gathered}
\mathcal{I}_{m}\left(w_{m}, B_{r}\right) \leq \mathcal{I}_{m}\left(\zeta w+(1-\zeta) w_{m}, B_{r}\right)+o(1) \\
\leq \mathcal{I}_{m}\left(w, B_{r}\right)+c \mu_{m}\left(\overline{B_{r} \backslash B_{s}}\right)+c\left\|w-w_{m}\right\|_{L^{2}}^{2}\|D \zeta\|_{L^{\infty}}^{2}+o(1) .
\end{gathered}
$$

By Lebesgue's dominated convergence

$$
\mathcal{I}_{m}\left(w, B_{r}\right) \rightarrow \mathcal{I}_{0}\left(w, B_{r}\right),
$$

and so we infer by (2.12) and (2.14) that

$$
\limsup _{m \rightarrow \infty} \mathcal{I}_{m}\left(w_{m}, B_{r}\right) \leq \mathcal{I}_{0}\left(w, B_{r}\right)+c \mu\left(\overline{\left(B_{r} \backslash B_{s}\right.}\right) .
$$


Bearing in mind that $\mu\left(\overline{B_{r} \backslash B_{s}}\right) \rightarrow \mu\left(\partial B_{r}\right)=0$ in the limit as $s \rightarrow r-$, and using the notation (2.23) and (2.29), we finally obtain

$$
\left.\limsup _{m \rightarrow \infty} \mathcal{G}_{r}\left(Y_{m}, w_{m}\right) \leq \mathcal{G}_{r}\left(Y_{0}, w\right) \quad \text { for a.e. } r \in\right] 0,1[.
$$

Remark. - The above method works also for $q>2$. Since we do not know that $w \in W^{1, q}$, we would set $\varphi=\zeta\left(\tilde{w}_{k}-w_{m}\right)$ in (2.21), where the smooth functions $\tilde{w}_{k} \rightarrow w$ in $W^{1,2}$ as $k \rightarrow \infty$.

We next apply the following lemma, whose hypotheses are satisfied by (3.1), (2.27), (2.12) and (3.2). Thus we conclude (2.17).

Lemma 3.1. - Let $\mathfrak{Y}$ be a closed subset of $\mathbb{R}^{s}$. Let $G(Y)$ be a symmetric bilinear form on $\mathbb{R}^{N \times n}$ depending continuously on $Y \in \mathfrak{Y}$ and satisfying

$$
G(Y) \cdot(\zeta, \zeta) \geq \gamma|\zeta|^{2}, \quad|G(Y)| \leq \Gamma
$$

for some $0<\gamma \leq \Gamma<\infty$, and all $Y \in \mathfrak{Y}, \zeta \in \mathbb{R}^{N \times n}$.

Define the corresponding quadratic functional $\mathcal{G}_{r}$, with $0<r<1$, by

$$
\mathcal{G}_{r}(Y, w)=\int_{B_{r}} G(Y) \cdot(D w, D w) d z
$$

for $Y \in L^{2}(B, \mathfrak{Y})$ and $w \in W^{1,2}\left(B, \mathbb{R}^{N}\right)$.

Suppose that

(i) $Y_{m} \rightarrow Y_{0}$ in $L^{2}(B, \mathfrak{Y})$,

(ii) $w_{m} \rightarrow w$ weakly in $W^{1,2}\left(B, \mathbb{R}^{N}\right)$,

(iii) $\limsup \mathcal{G}_{r}\left(Y_{m}, w_{m}\right) \leq \mathcal{G}_{r}\left(Y_{0}, w\right)$ for a.e. $\left.r \in\right] 0,1[$.

Then

$$
D w_{m} \rightarrow D w \quad \text { in } L_{\mathrm{loc}}^{2}\left(B, \mathbb{R}^{N \times n}\right) .
$$

Proof. - The assumption (i), together with the continuity of $G$, implies that $G\left(Y_{m}(z)\right) \rightarrow G\left(Y_{0}(z)\right)$ for a.e. $z \in B$. Hence, by Lebesgue's dominated convergence, we conclude that

$$
G\left(Y_{m}\right) \cdot D w \rightarrow G\left(Y_{0}\right) \cdot D w \quad \text { in } L^{2}\left(B, \mathbb{R}^{N \times n}\right) .
$$

Vol. 13, n $3-1996$. 
Now we fix some $r \in] 0,1[$ such that (iii) holds. Then we have

$$
\begin{aligned}
\gamma \underset{m \rightarrow \infty}{\limsup } \int_{B_{r}}\left|D w_{m}-D w\right|^{2} d z \\
\leq \limsup _{m \rightarrow \infty} \int_{B_{r}} G\left(Y_{m}\right) \cdot\left(D w_{m}-D w, D w_{m}-D w\right) d z \\
\leq \limsup _{m \rightarrow \infty} \int_{B_{r}} G\left(Y_{m}\right) \cdot\left(D w_{m}, D w_{m}\right) d z \\
\quad+\lim _{m \rightarrow \infty} \int_{B_{r}} G\left(Y_{m}\right) \cdot(D w, D w) d z \\
\quad-2 \lim _{m \rightarrow \infty} \int_{B_{r}} G\left(Y_{m}\right) \cdot\left(D w, D w_{m}\right) d z \\
\leq(1+1-2) \int_{B_{r}} G\left(Y_{0}\right) \cdot(D w, D w) d z=0 .
\end{aligned}
$$

We have used (iii) for the first term, and (3.5) and (ii) for the second and third terms. Therefore

$$
\lim _{m \rightarrow \infty} \int_{B_{r}}\left|D w_{m}-D w\right|^{2} d z=0
$$

and we have proved (3.4).

The one remaining task is to show (2.15). By virtue of (3.4) and Lebesgue's modified convergence, we can now proceed to the limit as $m \rightarrow \infty$ in the inequality $(2.21)$, for $\varphi \in C_{0}^{\infty}\left(B, \mathbb{R}^{N}\right)$. In this manner we deduce that

$$
\mathcal{I}_{0}(w, \operatorname{supp} \varphi) \leq \mathcal{I}_{0}(w+\varphi, \operatorname{supp} \varphi) \text { for every } \varphi \in C_{0}^{\infty}\left(B, \mathbb{R}^{N}\right),
$$

i.e. $w \in W^{1,2}\left(B, \mathbb{R}^{N}\right)$ is a minimizer for the functional $\mathcal{I}_{0}$. It follows that $w$ is a weak solution of the Euler equation associated with $\mathcal{I}_{0}$ :

$$
\operatorname{div}\left(F_{P P}\left(x_{0}, u_{0}, P_{0}\right) \cdot D w\right)=0 .
$$

This finishes the proof of Theorem 1.2.

Example 3.1. - The following example illustrates that Lemma 3.1 becomes false if we weaken strict positivity of $G(Y)$ to nonnegativity and strict rank-one-positivity

$$
G(Y) \cdot(\zeta, \zeta) \geq 0 \quad \text { and } \quad G(Y) \cdot(\eta \otimes \xi, \eta \otimes \xi) \geq \gamma|\eta \otimes \xi|^{2},
$$

for all $Y \in \mathfrak{Y}$ and $\zeta \in \mathbb{R}^{N \times n}, \eta \in \mathbb{R}^{N}, \xi \in \mathbb{R}^{n}$.

We let $n=N=2$ and we define the bilinear form

$$
G(Y) \cdot(\zeta, \zeta)=|\zeta|^{2}-2 Y \operatorname{det} \zeta,
$$


for $Y \in \mathfrak{Y}=[-1,1], \zeta \in \mathbb{R}^{2 \times 2}$, which is clearly nonnegative but strictly rank-one-positive. We define the functions $w_{m} \in W^{1,2}\left(B, \mathbb{R}^{2}\right)$ on the unit ball $B \subset \mathbb{R}^{2}$ by

$$
w_{m}(x)=r_{m}^{-1} \varphi_{m}(r) x
$$

for a sequence of numbers $r_{m} \rightarrow 0$, where $r=|x|$ and

$$
\varphi_{m}(r)=\left\{\begin{array}{cl}
1 & \text { for } r \leq r_{m} \\
\left(r_{m} / r\right)^{2} & \text { for } \quad r_{m}<r \leq 1
\end{array}\right.
$$

Then

$$
\int_{B}\left|D w_{m}\right|^{2} d x \rightarrow 4 \pi, \quad \int_{B}\left|w_{m}\right|^{2} d x \rightarrow 0,
$$

hence also $D w_{m} \rightarrow 0$ weakly in $L^{2}\left(B, \mathbb{R}^{2 \times 2}\right)$. We also define the functions $Y_{m} \in L^{2}(B, \mathfrak{Y})$ by

$$
Y_{m}(r)=\left\{\begin{array}{cl}
1 & \text { for } \quad r \leq r_{m} \\
-1 & \text { for } \quad r_{m}<r \leq 1
\end{array}\right.
$$

for which

$$
G\left(Y_{m}\right) \cdot\left(D w_{m}, D w_{m}\right)=0 \quad \text { and } \quad \int_{B}\left|Y_{m}+1\right|^{2} d x \rightarrow 0 .
$$

Remark. - Lemma 3.1 should be compared with [E-G2, Thm. 1]. Example 3.1 shows that this theorem does not generalize to uniformly strictly quasiconvex integrals of the form $\mathcal{I}(Y, w)=\int F(x, Y, D w) d x$ under the hypotheses that $Y_{m} \rightarrow Y$ in $L^{2}, w_{m} \rightarrow w$ weakly in $W^{1,2}$ and $\mathcal{I}\left(Y_{m}, w_{m}\right) \rightarrow \mathcal{I}(Y, w)$.

\section{THE GENERAL CASE}

Here we assume Hypotheses $\mathrm{H} 1, \mathrm{H} 2, \mathrm{H} 3$ and $\mathrm{H} 4$, with $q \geq 2$. We need a simple algebraic lemma, essentially proved in [E1, Lemma 5.2] or [G1, Lemma 3.1, p. 161], and the higher integrability theorem of F. W. Gehring, M. Giaquinta and G. Modica [G1, Prop. 1.1, p. 122].

LEMMA 4.1. - Let $f:\left[\frac{1}{2} r, r\right] \rightarrow[0, \infty[$ be bounded and satisfy

$$
f(t) \leq \theta f(s)+A(s-t)^{-2}+B(s-t)^{-q}+C
$$

for $\frac{1}{2} r \leq t<s \leq r$, where $A, B, C$ are nonnegative constants and $0 \leq \theta<1$. 
Then there exists a constant $c=c(\theta, q)$ such that

$$
f\left(\frac{1}{2} r\right) \leq c\left(A r^{-2}+B r^{-q}+C\right)
$$

THEOREM 4.1. - Let $\Omega$ be a bounded open set in $\mathbb{R}^{n}$, and let $g \in L_{\text {loc }}^{1}(\Omega)$ and $f \in L_{\mathrm{loc}}^{t}(\Omega)$ be nonnegative functions with $0<s<1<t<\infty$. Suppose we have

$$
f_{B_{\frac{1}{2} r}\left(x_{0}\right)} g d x \leq b\left(f_{B_{r}\left(x_{0}\right)} g^{s} d x\right)^{1 / s}+f_{B_{r}\left(x_{0}\right)} f d x
$$

for every ball $B_{r}\left(x_{0}\right) \subset \subset \Omega$. Then $g \in L_{\text {loc }}^{1+\varepsilon}(\Omega)$ for $\varepsilon<\varepsilon_{0}$, and

$$
\left(f_{B_{\varrho}\left(x_{0}\right)} g^{1+\varepsilon} d x\right)^{1 /(1+\varepsilon)} \leq c f_{B_{r}\left(x_{0}\right)} g d x+c\left(f_{B_{r}\left(x_{0}\right)} f^{1+\varepsilon} d x\right)^{1 /(1+\varepsilon)}
$$

for any ball $B_{r}\left(x_{0}\right) \subset \subset \Omega$ and $0<\varrho<r$, where $\varepsilon_{0}=\varepsilon_{0}(n, s, t, b)$ and $c=c\left(n, s, t, b, \frac{\varrho}{r}, \varepsilon\right)$.

The next theorem is proved in [G3, Prop. 2.2 and (2.18)], $c f$. [G-G1, Thm. 4.1].

THEOREM 4.2. - Let $u \in W^{1, q}\left(\Omega, \mathbb{R}^{N}\right)$ be a minimizer for the variational integral $\mathcal{I}$, whose integrand $F$ satisfies Hypotheses $H 1$ and $H 4$.

Then $D u \in L_{\text {loc }}^{q\left(1+\varepsilon^{\prime}\right)}\left(\Omega, \mathbb{R}^{N \times n}\right)$ for $\varepsilon^{\prime}<\varepsilon_{1}$, and

$$
\left(f_{B_{r}\left(x_{0}\right)}\left(1+|D u|^{q}\right)^{1+\varepsilon^{\prime}} d x\right)^{1 /\left(1+\varepsilon^{\prime}\right)} \leq c f_{B_{R}\left(x_{0}\right)}\left(1+|D u|^{q}\right) d x
$$

for any ball $B_{R}\left(x_{0}\right) \subset \subset \Omega$ and $0<r<R$, where $\varepsilon_{1}=\varepsilon_{1}\left(n, N, q, \frac{\Gamma}{\gamma}\right)$ and $c=c\left(n, N, q, \frac{\Gamma}{\gamma}, \frac{r}{R}, \varepsilon^{\prime}\right)$.

The corollary to the following theorem provides a uniform bound in $L_{\text {loc }}^{2(1+\varepsilon)}$ for the gradients of the blow-up functions $w_{m}$.

THEOREM 4.3. - Let $u \in W^{1, q}\left(\Omega, \mathbb{R}^{N}\right)$ be a minimizer for the variational integral $\mathcal{I}$, whose integrand $F$ satisfies Hypotheses $\mathrm{H1}, \mathrm{H} 2, \mathrm{H} 3$ and H4. Let

$$
\alpha_{0}=\frac{\varepsilon_{1}}{1+\varepsilon_{1}} \frac{\delta}{2}
$$

where $\varepsilon_{1}$ is the higher integrability constant of Theorem 4.2. 
Then, for every $0<\alpha<\alpha_{0}$, there exist positive constants $\varepsilon$ and $c\left(\frac{\varrho}{R}\right)$ such that

$$
\begin{aligned}
& \left(f_{B_{\varrho}\left(x_{0}\right)}\left(\left|D u-P_{0}\right|^{2}+\left|D u-P_{0}\right|^{q}\right)^{1+\varepsilon} d x\right)^{1 /(1+\varepsilon)} \\
& \leq c f_{B_{R}\left(x_{0}\right)}\left(\left|D u-P_{0}\right|^{2}+\left|D u-P_{0}\right|^{q}\right) d x \\
& \quad+c R^{2 \alpha}\left(f_{B_{R}\left(x_{0}\right)}\left(1+|D u|^{q}\right) d x\right)^{1+2 \alpha / q}
\end{aligned}
$$

holds for any ball $B_{R}\left(x_{0}\right) \subset \subset \Omega$ and $P_{0} \in \mathbb{R}^{N \times n}$, with $\left|u_{x_{0}, R}\right| \leq L$ and $\left|P_{0}\right| \leq L$, and $0<\varrho<R$.

COROllary 4.1. - In terms of $w_{m} \in W^{1, q}\left(B, \mathbb{R}^{N}\right)$, we have, for $0<r<1$,

$$
\left(f_{B_{r}}\left(\left|D w_{m}\right|^{2}+\lambda_{m}^{q-2}\left|D w_{m}\right|^{q}\right)^{1+\varepsilon} d z\right)^{1 /(1+\varepsilon)} \leq c(r) .
$$

Proof of Corollary 4.1. - Substituting (2.4), $x_{0}=x_{m}, R=r_{m}, \varrho=r r_{m}$ and $P_{0}=P_{m}$ in Theorem 4.3, and using (2.6) and (2.9) yields

$$
\begin{aligned}
& \left(f_{B_{r}}\left(\left|D w_{m}\right|^{2}+\lambda_{m}^{q-2}\left|D w_{m}\right|^{q}\right)^{1+\varepsilon} d z\right)^{1 /(1+\varepsilon)} \\
& \quad \leq c(r) f_{B}\left(\left|D w_{m}\right|^{2}+\lambda_{m}^{q-2}\left|D w_{m}\right|^{q}\right) d z \\
& \quad+c(r) \lambda_{m}^{-2} r_{m}^{2 \alpha}\left(f_{B}\left(1+\left|P_{m}+\lambda_{m} D w_{m}\right|^{q}\right) d z\right)^{1+2 \alpha / q} \leq c(r) .
\end{aligned}
$$

Proof of Theorem 4.3. - We fix $B_{R}\left(x_{0}\right) \subset \subset \Omega$ and $P_{0} \in \mathbb{R}^{N \times n}$, subject to the conditions $\left|u_{0}\right| \leq L$ and $\left|P_{0}\right| \leq L$, where $u_{0}=u_{x_{0}, R}$. Then, for $(x, u, P) \in \bar{\Omega} \times \mathbb{R}^{N} \times \mathbb{R}^{N \times n}$, we set

$$
\bar{F}(x, u, P)=F\left(x, u, P_{0}+P\right)-F\left(x, u, P_{0}\right)-F_{P}\left(x, u, P_{0}\right) \cdot P .
$$

Clearly $\bar{F}$ is strictly quasiconvex, and $\bar{F}(x, u, 0)=0$. Moreover, we have the estimates

$$
\begin{gathered}
|\bar{F}(x, u, P)| \leq c\left(|P|^{2}+|P|^{q}\right) \\
\left|\bar{F}_{P}(x, u, P)\right| \leq c\left(|P|+|P|^{q-1}\right), \\
|\bar{F}(x, u, P+Q)-\bar{F}(x, u, P)| \leq c\left(|P|+|P|^{q-1}+|Q|+|Q|^{q-1}\right)|Q| .
\end{gathered}
$$


We now fix some ball $B_{r}\left(y_{0}\right) \subset \subset B_{R}\left(x_{0}\right)$. We let $\frac{1}{2} r \leq t<s \leq r$, and we let $\zeta \in C_{0}^{\infty}\left(B_{s}\left(y_{0}\right)\right)$ be a cut-off function with $0 \leq \zeta \leq 1, \zeta \equiv 1$ on $B_{t}\left(y_{0}\right)$ and $|D \zeta| \leq c(s-t)^{-1}$. We set

$$
P(x)=u_{0}+P_{0} \cdot\left(x-y_{0}\right), \quad \varphi=\zeta(u-P), \quad \psi=(1-\zeta)(u-P),
$$

for which

$$
\varphi+\psi=u-P, \quad D \varphi+D \psi=D u-P_{0} .
$$

The strict quasiconvexity of $\bar{F}$ at 0 asserts that

$$
\begin{aligned}
(4.1) \gamma & \int_{B_{s}}\left(|D \varphi|^{2}+|D \varphi|^{q}\right) d x \leq \int_{B_{s}} \bar{F}\left(y_{0}, u_{0}, D \varphi\right) d x \\
= & \int_{B_{s}} \bar{F}\left(y_{0}, u_{0}, D u-P_{0}-D \psi\right) d x \\
= & \int_{B_{s}} \bar{F}\left(y_{0}, u_{0}, D u-P_{0}\right) d x \\
& +\int_{B_{s}}\left(\bar{F}\left(y_{0}, u_{0}, D u-P_{0}-D \psi\right)-\bar{F}\left(y_{0}, u_{0}, D u-P_{0}\right)\right) d x \\
\leq & \int_{B_{s}} \bar{F}\left(y_{0}, u_{0}, D u-P_{0}\right) d x \\
& +c \int_{B_{s} \backslash B_{t}}\left(\left|D u-P_{0}\right|+\left|D u-P_{0}\right|^{q-1}+|D \psi|+|D \psi|^{q-1}\right)|D \psi| d x .
\end{aligned}
$$

On the other hand

$$
\begin{aligned}
& \int_{B_{s}} \bar{F}\left(y_{0}, u_{0}, D u-P_{0}\right) d x \\
& =\int_{B_{s}} \int_{0}^{1} F_{P}\left(y_{0}, u_{0}, s D u+(1-s) P_{0}\right) \cdot\left(D u-P_{0}\right) d s d x \\
& \quad-\int_{B_{s}} F_{P}\left(y_{0}, u_{0}, P_{0}\right) \cdot\left(D u-P_{0}\right) d x \\
& =\int_{B_{s}} F(x, u, D u) d x-\int_{B_{s}} F\left(x, u, P_{0}\right) d x \\
& \quad+\int_{B_{s}} \int_{0}^{1}\left(F_{P}\left(y_{0}, u_{0}, s D u+(1-s) P_{0}\right)-F_{P}\left(x, u, s D u+(1-s) P_{0}\right)\right) \\
& \quad \times\left(D u-P_{0}\right) d s d x-\int_{B_{s}} F_{P}\left(y_{0}, u_{0}, P_{0}\right) \cdot D \psi d x,
\end{aligned}
$$


while from the minimality of $u$

$$
\begin{array}{rl}
\int_{B_{s}} & F(x, u, D u) d x \leq \int_{B_{s}} F(x, u-\varphi, D u-D \varphi) d x \\
= & \int_{B_{s}} F\left(x, P+\psi, P_{0}+D \psi\right) d x \\
= & \int_{B_{s}} \bar{F}(x, u, D \psi) d x+\int_{B_{s}} F\left(x, u, P_{0}\right) d x \\
& +\int_{B_{s}} F_{P}\left(x, u, P_{0}\right) \cdot D \psi d x \\
& +\int_{B_{s}}\left(F\left(x, P+\psi, P_{0}+D \psi\right)-F\left(x, u, P_{0}+D \psi\right)\right) d x
\end{array}
$$

Combining (4.1), (4.2) and (4.3) we obtain

$$
\begin{aligned}
& \gamma \int_{B_{s}}\left(|D \varphi|^{2}+|D \varphi|^{q}\right) d x \leq \int_{B_{s}} \bar{F}(x, u, D \psi) d x \\
& \quad+\int_{B_{s}} \int_{0}^{1}\left(F_{P}\left(y_{0}, u_{0}, s D u+(1-s) P_{0}\right)-F_{P}\left(x, u, s D u+(1-s) P_{0}\right)\right) \\
& \quad \times\left(D u-P_{0}\right) d s d x \\
& \quad+c \int_{B_{s} \backslash B_{t}}\left(\left|D u-P_{0}\right|^{2}+\left|D u-P_{0}\right|^{q}\right) d x+c \int_{B_{s} \backslash B_{t}}\left(|D \psi|^{2}+|D \psi|^{q}\right) d x \\
& \quad+\int_{B_{s}}\left(F\left(x, P+\psi, P_{0}+D \psi\right)-F\left(x, u, P_{0}+D \psi\right)\right) d x \\
& \quad+\int_{B_{s}}\left(F_{P}\left(x, u, P_{0}\right)-F_{P}\left(y_{0}, u_{0}, P_{0}\right)\right) \cdot D \psi d x
\end{aligned}
$$

and further, by Hypothesis $\mathrm{H} 2$,

$$
\begin{aligned}
& \int_{B_{t}}\left(\left|D u-P_{0}\right|^{2}+\left|D u-P_{0}\right|^{q}\right) d x \\
& \leq c\left(\int_{B_{s} \backslash B_{t}}\left(\left|D u-P_{0}\right|^{2}+\left|D u-P_{0}\right|^{q}\right) d x\right. \\
& \quad+(s-t)^{-2} \int_{B_{r}}|u-P|^{2} d x+(s-t)^{-q} \int_{B_{r}}|u-P|^{q} d x \\
& \left.\quad+\int_{B_{r}} \omega\left(\left|u_{0}\right|, c\left|x-y_{0}\right|+\left|u-u_{0}\right|\right)\left(1+|D u|^{q}\right) d x\right) .
\end{aligned}
$$

We now fill the hole, that is, we add $c$ times the left hand side to both sides and we divide the resulting inequality by $1+c$. Setting $g=\left|D u-P_{0}\right|^{2}+\left|D u-P_{0}\right|^{q}, \quad f=\omega\left(L, c R+\left|u-u_{0}\right|\right)\left(1+|D u|^{q}\right)$, Vol. 13, $\mathrm{n}^{\circ}$ 3-1996. 
we thus obtain

$$
\begin{aligned}
\int_{B_{t}} g d x \leq \frac{c}{1+c}( & \int_{B_{s}} g d x+(s-t)^{-2} \int_{B_{r}}|u-P|^{2} d x \\
& \left.+(s-t)^{-q} \int_{B_{r}}|u-P|^{q} d x+\int_{B_{r}} f d x\right) .
\end{aligned}
$$

By means of Lemma 4.1, we deduce the following Caccioppoli inequality with error term

$\int_{B_{\frac{1}{2} r}} g d x \leq c r^{-2} \int_{B_{r}}|u-P|^{2} d x+c r^{-q} \int_{B_{r}}|u-P|^{q} d x+c \int_{B_{r}} f d x$.

Applying the Poincaré-Sobolev and Hölder inequalities results in

$$
\begin{aligned}
& f_{B_{\frac{1}{2} r}} g d x \leq c\left(f_{B_{r}}\left|D u-P_{0}\right|^{2 *} d x\right)^{2 / 2_{*}} \\
& \quad+c\left(f_{B_{r}}\left|D u-P_{0}\right|^{q_{*}} d x\right)^{q / q_{*}}+c f_{B_{r}} f d x \\
& \leq c\left(f_{B_{r}}\left(\left|D u-P_{0}\right|^{2}+\left|D u-P_{0}\right|^{q}\right)^{2 * / 2} d x\right)^{2 / 2_{*}}+c f_{B_{r}} f d x .
\end{aligned}
$$

Therefore, for $s=2_{*} / 2<1$, we have proved that

$$
f_{B_{\frac{1}{2} r}\left(y_{0}\right)} g d x \leq c\left(f_{B_{r}\left(y_{0}\right)} g^{s} d x\right)^{1 / s}+c f_{B_{r}\left(y_{0}\right)} f d x
$$

for all $B_{r}\left(y_{0}\right) \subset \subset B_{R}\left(x_{0}\right)$. Invoking Theorem 4.1 we finally arrive at

$$
\left(f_{B_{\varrho}\left(x_{0}\right)} g^{1+\varepsilon} d x\right)^{1 /(1+\varepsilon)} \leq c f_{B_{R}\left(x_{0}\right)} g d x+c\left(f_{B_{r}\left(x_{0}\right)} f^{1+\varepsilon} d x\right)^{1 /(1+\varepsilon)}
$$

for all $0<\varepsilon<\varepsilon_{0}, 0<\varrho<R$ and $r=\frac{1}{2}(\varrho+R)$. Here the constant $c$ also depends on $\frac{\varrho}{R}$ and $\varepsilon$.

For $\varepsilon<\varepsilon^{\prime}<\varepsilon_{1}$, where $\varepsilon_{1}$ is the constant from Theorem 4.2, we next set

$$
\alpha=\beta \frac{\delta}{2} \quad \text { with } \quad \beta=\frac{\varepsilon^{\prime}-\varepsilon}{\left(1+\varepsilon^{\prime}\right)(1+\varepsilon)} .
$$

(Since $\alpha \rightarrow \alpha_{0}$ as $\left(\varepsilon, \varepsilon^{\prime}\right) \rightarrow\left(0, \varepsilon_{1}\right)$ and $\alpha \rightarrow 0$ as $\varepsilon^{\prime}-\varepsilon \rightarrow 0$, any value $\alpha \in] 0, \alpha_{0}$ [ is attained in this manner.) Then, using the Hölder, Jensen and 
Poincare inequalities, the boundedness and concavity of $\omega(L, \cdot)$, Theorem 4.2 and the estimate $\omega(L, t) \leq c t^{\delta}$, we control the last term of (4.4) by

$$
\begin{aligned}
& \left(f_{B_{r}} f^{1+\varepsilon} d x\right)^{1 /(1+\varepsilon)} \\
& \quad \leq c\left(f_{B_{r}} \omega\left(L, c R+\left|u-u_{0}\right|\right) d x\right)^{\beta}\left(f_{B_{r}}\left(1+|D u|^{q}\right)^{1+\varepsilon^{\prime}} d x\right)^{1 /\left(1+\varepsilon^{\prime}\right)} \\
& \quad \leq c \omega\left(L, c R+f_{B_{R}}\left|u-u_{0}\right| d x\right)^{\beta} f_{B_{R}}\left(1+|D u|^{q}\right) d x \\
& \quad \leq c \omega\left(L, c R\left(f_{B_{R}}\left(1+|D u|^{q}\right) d x\right)^{1 / q}\right)^{\beta} f_{B_{R}}\left(1+|D u|^{q}\right) d x \\
& \quad \leq c R^{2 \alpha}\left(f_{B_{R}}\left(1+|D u|^{q}\right) d x\right)^{1+2 \alpha / q} \cdot \square
\end{aligned}
$$

Lemma 4.2. - The function $w \in W^{1,2}\left(B, \mathbb{R}^{N}\right)$ is a weak solution of the linear elliptic system

$$
\operatorname{div}\left(F_{P P}\left(x_{0}, u_{0}, P_{0}\right) \cdot D w\right)=0 .
$$

In particular, we conclude that $w \in C^{\infty}\left(B, \mathbb{R}^{N}\right)$.

Proof. - Since $Y \rightarrow G(Y)$ is continuous at the point $Y_{0} \in \mathfrak{Y}$ and satisfies (2.22), there exists a continuous, bounded and concave function $\chi(t)$, with $\chi(0)=0$, such that

$$
\left|G(Y)-G\left(Y_{0}\right)\right| \leq \chi\left(\left|Y-Y_{0}\right|^{2}\right)\left(1+|Q|^{q-2}\right)
$$

for all $Y=(x, u, P, Q) \in \mathfrak{Y}$.

We let $\varphi \in C_{0}^{\infty}\left(B, \mathbb{R}^{N}\right)$ and we choose $\left.r \in\right] 0,1[$ such that supp $\varphi \subset \subset B_{r}$. We then write (2.21) as

$$
\mathcal{G}_{r}\left(Y_{m}, w_{m}\right) \leq \mathcal{G}_{r}\left(\bar{Y}_{m}, w_{m}+\varphi\right)+o(1),
$$

using (2.29), (2.24) and the functions

$$
\bar{Y}_{m}=Y_{m}+\left(0, r_{m} \lambda_{m} \varphi, 0, \lambda_{m} D \varphi\right) \in L^{q}(B, \mathfrak{Y}) .
$$

By (2.27), we observe that

$$
\bar{Y}_{m} \rightarrow Y_{0} \quad \text { in } L^{2}(B, \mathfrak{Y})
$$

Vol. 13, n 3-1996. 
Clearly (4.6) is equivalent to

$$
\begin{aligned}
0 \leq & 2 \int_{B_{r}} G\left(\bar{Y}_{m}\right) \cdot\left(D w_{m}, D \varphi\right) d z+\int_{B_{r}} G\left(\bar{Y}_{m}\right) \cdot(D \varphi, D \varphi) d z \\
& +\int_{B_{r}}\left(G\left(\bar{Y}_{m}\right)-G\left(Y_{m}\right)\right) \cdot\left(D w_{m}, D w_{m}\right) d z+o(1) \\
\leq & 2 \int_{B_{r}} G\left(Y_{0}\right) \cdot\left(D w_{m}, D \varphi\right) d z+\int_{B_{r}} G\left(Y_{0}\right) \cdot(D \varphi, D \varphi) d z \\
& +c \int_{B_{r}}\left|G\left(\bar{Y}_{m}\right)-G\left(Y_{0}\right)\right|\left(1+\left|D w_{m}\right|^{2}\right) d z \\
& +\int_{B_{r}}\left|G\left(Y_{m}\right)-G\left(Y_{0}\right)\right|\left|D w_{m}\right|^{2} d z+o(1) \\
= & (\mathrm{I})+(\mathrm{II})+(\mathrm{III})+(\mathrm{IV})+o(1) .
\end{aligned}
$$

On account of (4.5), we estimate the term (III) as

$$
\begin{aligned}
(\mathrm{III}) \leq & c(r) f_{B_{r}} \chi\left(\left|\bar{Y}_{m}-Y_{0}\right|^{2}\right)\left(1+\left|D w_{m}\right|^{2}+\lambda_{m}^{q-2}\left|D w_{m}\right|^{q}\right) d z \\
\leq & c\left(f_{B_{r}} \chi\left(\left|\bar{Y}_{m}-Y_{0}\right|^{2}\right) d z\right)^{\varepsilon /(1+\varepsilon)} \\
& \times\left(f_{B_{r}}\left(1+\left|D w_{m}\right|^{2}+\lambda_{m}^{q-2}\left|D w_{m}\right|^{q}\right)^{1+\varepsilon} d z\right)^{1 /(1+\varepsilon)} \\
\leq & c(r) \chi\left(f_{B_{r}}\left|\bar{Y}_{m}-Y_{0}\right|^{2} d z\right)^{\varepsilon /(1+\varepsilon)}=o(1) .
\end{aligned}
$$

Here we have used the Hölder and Jensen inequalities, the boundedness and concavity of $\chi(t)$, Corollary 4.1 and the limit (4.7). The term (IV) is estimated in the same way. Therefore, by (2.12), we infer from (4.8) that

$$
0 \leq 2 \int_{B_{r}} G\left(Y_{0}\right) \cdot(D w, D \varphi) d z+\int_{B_{r}} G\left(Y_{0}\right) \cdot(D \varphi, D \varphi) d z .
$$

This implies that

$$
\int_{B} G\left(Y_{0}\right) \cdot(D w, D \varphi) d z=0 \text { for every } \varphi \in C_{0}^{\infty}\left(B, \mathbb{R}^{N}\right) .
$$

LEMMA 4.3. - We have the limits

$$
D w_{m} \rightarrow D w \quad \text { in } L_{\mathrm{loc}}^{2}\left(B, \mathbb{R}^{N \times n}\right),
$$




$$
\lambda_{m}^{(q-2) / q} D w_{m} \rightarrow 0 \quad \text { in } L_{\text {loc }}^{q}\left(B, \mathbb{R}^{N \times n}\right) \quad(\text { for } q>2) .
$$

In the proof we shall make frequent use of the fact that the function $w$ and its gradient $D w$ are bounded on compact subsets of $B$.

We fix some $r \in] 0,1\left[\right.$ with $\mu\left(\partial B_{r}\right)=0$ (recall that a.e. $\left.r \in\right] 0,1$ [ satisfies this condition). We let $0<s<r$ and $\zeta \in C_{0}^{\infty}\left(B_{r}\right)$ be a cut-off function with $0 \leq \zeta \leq 1$ and $\zeta \equiv 1$ on $B_{s}$. Setting $\varphi=\zeta\left(w-w_{m}\right) \in W_{0}^{1, q}\left(B, \mathbb{R}^{N}\right)$ in (2.21) and using (2.20) and (2.13) yields

$$
\begin{aligned}
& \mathcal{I}_{m}\left(w_{m}, B_{r}\right) \leq \mathcal{I}_{m}\left(\zeta w+(1-\zeta) w_{m}, B_{r}\right)+o(1) \\
& \quad \leq \mathcal{I}_{m}\left(w, B_{r}\right)+c \mu_{m}\left(\overline{B_{r} \backslash B_{s}}\right)+c\left\|w-w_{m}\right\|_{L^{2}}^{2}\|D \zeta\|_{L^{\infty}}^{2} \\
& \quad+c \lambda_{m}^{q-2}\left\|w-w_{m}\right\|_{L^{q}\left(B_{r}\right)}^{q}\|D \zeta\|_{L^{\infty}}^{q}+o(1) .
\end{aligned}
$$

Since by Lebesgue's dominated convergence

$$
\mathcal{I}_{m}\left(w, B_{r}\right) \rightarrow \mathcal{I}_{0}\left(w, B_{r}\right),
$$

it follows by recourse to (2.12) and (2.14) that

$$
\limsup _{m \rightarrow \infty} \mathcal{I}_{m}\left(w_{m}, B_{r}\right) \leq \mathcal{I}_{0}\left(w, B_{r}\right)+c \mu\left(\overline{B_{r} \backslash B_{s}}\right) .
$$

Letting $s \rightarrow r-$, so that $\mu\left(\overline{B_{r} \backslash B_{s}}\right) \rightarrow \mu\left(\partial B_{r}\right)=0$, we obtain by (2.29) that

$$
\limsup _{m \rightarrow \infty} \mathcal{G}_{r}\left(Y_{m}, w_{m}\right) \leq \mathcal{G}_{r}\left(Y_{0}, w\right) .
$$

According to Hypothesis H3, (2.19) and (2.24), we have

$$
\begin{aligned}
& \gamma \int_{B}\left(|D \varphi|^{2}+\lambda_{m}^{q-2}|D \varphi|^{q}\right) d z \leq \int_{B} F_{m}(0,0, D \varphi) d z \\
& \quad=\int_{B} G\left(\tilde{Y}_{m}\right) \cdot(D \varphi, D \varphi) d z
\end{aligned}
$$

for $\varphi \in W_{0}^{1, q}\left(B, \mathbb{R}^{N}\right)$ and $\tilde{Y}_{m}=\left(x_{m}, u_{m}, P_{m}, \lambda_{m} D \varphi\right) \in L^{q}(B, \mathfrak{Y})$. Here we set $\varphi=\zeta\left(w_{m}-w\right)$, where $\zeta \in C_{0}^{\infty}\left(B_{r}\right)$ is the same cut-off function as before, for $0<s<r$. Then

$$
\tilde{Y}_{m}=\left(x_{m}, u_{m}, P_{m}, \lambda_{m} \zeta\left(D w_{m}-D w\right)+\lambda_{m}\left(w_{m}-w\right) \otimes D \zeta\right),
$$


for which we note, by (2.12), the limit

$$
\tilde{Y}_{m} \rightarrow Y_{0} \quad \text { in } L^{2}(B, \mathfrak{Y})
$$

We consider the difference

$$
\begin{aligned}
& \mathcal{G}_{r}\left(\tilde{Y}_{m}, w_{m}\right)-\mathcal{G}_{r}\left(Y_{m}, w_{m}\right)=\int_{B_{r}}\left(G\left(\tilde{Y}_{m}\right)-G\left(Y_{0}\right)\right) \cdot\left(D w_{m}, D w_{m}\right) d z \\
& \quad+\int_{B_{r}}\left(G\left(Y_{0}\right)-G\left(Y_{m}\right)\right) \cdot\left(D w_{m}, D w_{m}\right) d z=(\mathrm{I})+(\mathrm{II}) .
\end{aligned}
$$

On account of (4.5), the Hölder and Jensen inequalities, the boundedness and concavity of $\chi(t),(4.13)$, Corollary 4.1, (2.12) and (2.9), we estimate the term (I) as

$$
\begin{aligned}
(\mathrm{I}) \leq & c(r) f_{B_{r}} \chi\left(\left|\tilde{Y}_{m}-Y_{0}\right|^{2}\right) \\
& \times\left(1+\lambda_{m}^{q-2}\left|D w_{m}-D w\right|^{q-2}+\lambda_{m}^{q-2}\left|w_{m}-w\right|^{q-2}|D \zeta|^{q-2}\right)\left|D w_{m}\right|^{2} d z \\
\leq & c \chi\left(f_{B_{r}}\left|\tilde{Y}_{m}-Y_{0}\right|^{2} d z\right)^{\varepsilon /(1+\varepsilon)} \\
& \times\left(f_{B_{r}}\left(\left|D w_{m}\right|^{2}+\lambda_{m}^{q-2}\left|D w_{m}\right|^{q}\right)^{1+\varepsilon} d z\right)^{1 /(1+\varepsilon)} \\
& +c\|D \zeta\|_{L^{\infty}}^{q-2}\left(\lambda_{m}^{(q-2) / q}\left\|w_{m}-w\right\|_{L^{q}\left(B_{r}\right)}\right)^{q-2}\left(\lambda_{m}^{(q-2) / q}\left\|D w_{m}\right\|_{L^{q}}\right)^{2} \\
= & o(1),
\end{aligned}
$$

and similarly for the term (II). Hence we conclude from (4.11) and (4.14) that also

$$
\limsup _{m \rightarrow \infty} \mathcal{G}_{r}\left(\tilde{Y}_{m}, w_{m}\right) \leq \mathcal{G}_{r}\left(Y_{0}, w\right)
$$

We now insert $\varphi=\zeta\left(w_{m}-w\right)$ in (4.12). By (2.22), (2.13) and (2.12), 
this gives

$$
\begin{aligned}
\gamma & \int_{B_{r}}\left(\left|D w_{m}-D w\right|^{2}+\lambda_{m}^{q-2}\left|D w_{m}-D w\right|^{q}\right) d z \\
\leq & \int_{B_{r}} G\left(\tilde{Y}_{m}\right) \cdot\left(D w_{m}-D w, D w_{m}-D w\right) d z \\
& +c \mu_{m}\left(\overline{B_{r} \backslash B_{s}}\right)+o(1) \\
\leq & \int_{B_{r}} G\left(\tilde{Y}_{m}\right) \cdot\left(D w_{m}, D w_{m}\right) d z+\int_{B_{r}} G\left(Y_{0}\right) \cdot(D w, D w) d z \\
& -2 \int_{B_{r}} G\left(Y_{0}\right) \cdot\left(D w, D w_{m}\right) d z \\
& +c \int_{B_{r}}\left|G\left(\tilde{Y}_{m}\right)-G\left(Y_{0}\right)\right|\left(1+\left|D w_{m}\right|\right) d z+c \mu_{m}\left(\overline{B_{r} \backslash B_{s}}\right)+o(1) \\
= & (\mathrm{i})+(\mathrm{ii})+(\mathrm{iii})+(\mathrm{iv})+(\mathrm{v})+o(1) .
\end{aligned}
$$

The estimate for the term (iv) is now routine.

$$
\begin{aligned}
\text { (iv) } \leq & c(r) f_{B_{r}} \chi\left(\left|\tilde{Y}_{m}-Y_{0}\right|^{2}\right) \\
& \times\left(1+\lambda_{m}^{q-2}\left|D w_{m}\right|^{q-2}+\lambda_{m}^{q-2}\left|w_{m}-w\right|^{q-2}|D \zeta|^{q-2}\right)\left(1+\left|D w_{m}\right|\right) d z \\
= & o(1) .
\end{aligned}
$$

Thus we infer from (4.16), using (4.15), (2.12) and (2.14), that

$$
\gamma \limsup _{m \rightarrow \infty} \int_{B_{r}}\left(\left|D w_{m}-D w\right|^{2}+\lambda_{m}^{q-2}\left|D w_{m}-D w\right|^{q}\right) d z \leq c \mu\left(\overline{B_{r} \backslash B_{s}}\right) .
$$

Since $\mu\left(\overline{B_{r} \backslash B_{s}}\right) \rightarrow \mu\left(\partial B_{r}\right)=0$ as $s \rightarrow r-$, we conclude that

$$
\lim _{m \rightarrow \infty} \int_{B_{r}}\left|D w_{m}-D w\right|^{2} d z=0, \quad \lim _{m \rightarrow \infty} \lambda_{m}^{q-2} \int_{B_{r}}\left|D w_{m}-D w\right|^{q} d z=0 .
$$

The last equation implies

$$
\lim _{m \rightarrow \infty} \lambda_{m}^{q-2} \int_{B_{r}}\left|D w_{m}\right|^{q} d z=0
$$

and we have shown that (4.9) and (4.10) hold.

In view of Lemmas 4.2 and 4.3, the proof of Theorem 1.1 is now complete. 


\section{REFERENCES}

[A-F] E. ACERBI and N. FusCo, A regularity theorem for minimizers of quasiconvex integrals. Arch. Rational Mech. Anal., Vol. 99, 1987, pp. 261-281.

[E1] L. C. EvANS, Quasiconvexity and partial regularity in the calculus of variations. Arch. Rational Mech. Anal., Vol. 95, 1986, pp. 227-252.

[E2] L. C. EvANS, Weak convergence methods for nonlinear partial differential equations. Regional conference series in mathematics, Vol. 74, AMS, Providence, 1990.

[E-G1] L. C. Evans and R. F. GARIEPY, Blow-up, compactness and partial regularity in the calculus of variations. Indiana Univ. Math. J., Vol. 36, 1987, pp. 361-371.

[E-G2] L. C. Evans and R. F. GARIEPY, Some remarks concerning quasiconvexity and strong convergence. Proc. Royal Soc. Edinburgh, Vol. 106A, 1987, pp. 53-61.

[F-H] N. Fusco and J. Hutchinson, $C^{1, \alpha}$ partial regularity of functions minimising quasiconvex integrals. Manuscripta math., Vol. 54, 1985, pp. 121-143.

[G1] M. GiaQuinta, Multiple integrals in the calculus of variations and nonlinear elliptic systems. Princeton Univ. Press, Princeton, 1983.

[G2] M. GIAQUINTA, The problem of the regularity of minimizers. International congress of mathematicians, Berkeley, 1986.

[G3] M. GIAQUINTA, Quasiconvexity, growth conditions and partial regularity. In: S. HiLDEBRANDT and R. LeIS (Eds.) Partial differential equations and calculus of variations. Lecture notes in mathematics, Vol. 1357, Springer, Berlin, 1988.

[G-G1] M. GiaQUINTA and E. GIUSTI, On the regularity of the minima of variational integrals. Acta Math., Vol. 148, 1982, pp. 31-46.

[G-G2] M. GiaquinTA and E. GiusTI, Differentiability of minima of nondifferentiable functionals. Inv. Math., Vol. 72, 1983, pp. 285-298.

[G-M] M. Giaquinta and G. Modica, Partial regularity of minimizers of quasiconvex integrals. Ann. Inst. H. Poincaré, Analyse non linéaire, Vol 3, 1986, pp. 185-208.

[H] C. HAMBURGER, An elementary partial regularity proof for solutions of nonlinear elliptic systems. SFB 256, Bonn, Preprint 353, 1994.

[Ho] M. C. HoNG, Existence and partial regularity in the calculus of variations. Ann. Mat. Pura Appl., Vol. 149, 1987, pp. 311-328.

[R] W. RuDIN, Real and complex analysis. McGraw-Hill, New York, 1987.

(Manuscript received June 21, 1994.) 